\title{
Clinical outcomes of hospitalised patients with catheter-associated urinary tract infection in countries with a high rate of multidrug-resistance: the COMBACTE- MAGNET RESCUING study
}

\author{
Aina Gomila ${ }^{1,2,3^{*}}$ (1), Jordi Carratalà ${ }^{1,4}$, Noa Eliakim-Raz ${ }^{5}$, Evelyn Shaw ${ }^{1}$, Cristian Tebé 6 , Martin Wolkewitz ${ }^{7}$ \\ Irith Wiegand ${ }^{8}$, Sally Grier ${ }^{9}$, Christiane Vank ${ }^{8}$, Nienke Cuperus $^{10}$, Leonard Van den Heuvel ${ }^{10}$, Cuong Vuong ${ }^{8}$, \\ Alasdair MacGowan ${ }^{9}$, Leonard Leibovici ${ }^{5}$, Ibironke Addy ${ }^{8}$, Miquel Pujol ${ }^{1}$ and on behalf of RESCUING Study Group \\ and Study Sites
}

\begin{abstract}
Background: Although catheter-associated urinary tract infection (CA-UTI) is a major healthcare-related problem worldwide, there is a scarcity of current data from countries with high antimicrobial resistance rates. We aimed to determine the clinical outcomes of patients with CA-UTI compared to those of patients with other sources of complicated urinary tract infection (CUTI), and to assess the impact of antimicrobial resistance. We also aimed to identify the factors influencing 30-day mortality among patients with CA-UTI.

Methods: This was a multicentre, multinational retrospective cohort study including hospitalised adults with cUTI between January 2013 and December 2014 in twenty hospitals from eight countries from southern Europe, Turkey and Israel. The primary endpoint was 30-day mortality. The secondary endpoints were length of hospital stay, symptom improvement after 7 days' treatment, symptom recurrence at 30 days and readmission 60 days after hospital discharge.

Results: Of the 807 CUTI episodes, 341 (42.2\%) were CA-UTIs. The time from catheter insertion to CUTI diagnosis was less than 2 weeks in $44.6 \%$ of cases. Overall, $74.5 \%$ of cases had hospital or healthcare-acquired CA-UTI. Compared to patients with other CUTI aetiologies, those with CA-UTI had the following characteristics: they were more frequently males, older, admitted for a reason other than CUTI and admitted from a long-term care facility; had higher Charlson's comorbidity index; and more frequently had polymicrobial infections and multidrug-resistant Gram-negative bacteria (MDR-GNB). Patients with CA-UTI also had significantly higher 30-day mortality rates (15.2\% vs 6\%) and longer hospital stay (median 14 [interquartile range -IQR- 7-27] days vs 8 [IQR 5-14] days) than patients with cUTI of other sources. After adjusting for confounders, CA-UTI was not independently associated with an increased risk of mortality (odds ratio, 1.40; 95\% confidence interval, 0.77-2.54), and neither was the presence of MDR-GNB.
\end{abstract}

(Continued on next page)

\footnotetext{
* Correspondence: agomilagrange@gmail.com

${ }^{1}$ Department of Infectious Diseases, Hospital Universitari de Bellvitge-Institut

d'Investigació Biomèdica de Bellvitge (IDIBELL), Institut Català de la Salut

(ICS-HUB), Barcelona, Spain

${ }^{2}$ Corporació Sanitària Parc Taulí, Barcelona, Spain

Full list of author information is available at the end of the article
}

(c) The Author(s). 2019 Open Access This article is distributed under the terms of the Creative Commons Attribution 4.0 International License (http://creativecommons.org/licenses/by/4.0/), which permits unrestricted use, distribution, and reproduction in any medium, provided you give appropriate credit to the original author(s) and the source, provide a link to the Creative Commons license, and indicate if changes were made. The Creative Commons Public Domain Dedication waiver (http://creativecommons.org/publicdomain/zero/1.0/) applies to the data made available in this article, unless otherwise stated. 
(Continued from previous page)

Conclusions: CA-UTI was the most frequent source of CUTI, affecting mainly frail patients. The mortality of patients with CA-UTI was high, though this was not directly related to the infection.

Keywords: Catheter-associated urinary tract infection, Antimicrobial resistance, Gram-negative infections

\section{Introduction}

Urinary tract infection (UTI) is a major healthcare problem worldwide. Catheter-associated UTI (CA-UTI) accounts for more than 1 million cases per year in the US [1]. These infections are also responsible for more than $80 \%$ of UTIs originating in the healthcare setting and are the most frequent cause of both bacteraemia in long-term care facilities (LTCF) and infection in patients with spinal cord injury [2, 3]. Approximately 20\% of hospitalised patients have a urinary catheter during admission, with the risk of CA-UTI increasing by $3-7 \%$ per day $[4,5]$.

Despite efforts to reduce the incidence of CA-UTI, rates continue to increase, with the US Centres for Disease Control and Prevention reporting a 6\% increase between 2009 and 2013 [6]. The high prevalence of CA-UTI is associated with significant antimicrobial use, and this not only favours the emergence and spread of multidrug-resistance (MDR) but also increases healthcare costs [7-9]. Antibiotic resistance, in particular, has become a major healthcare problem that complicates treatment and leads to worse outcomes. Even though these issues exist, CA-UTI is usually seen as a mild or lowrisk infection with no severe consequences for patients. However, most previous studies seeking to address this issue have only evaluated specific populations, such as critically ill patients, which limits extrapolation to other contexts. Thus, the question of whether CA-UTI development increases the risk of mortality remains unanswered, not least because conflicting results have previously been reported $[10,11]$.

We aimed to assess the clinical outcomes of CA-UTI among hospitalised patients with complicated UTI (cUTI) in a multicentre study of surgical and medical patients from eight countries with high prevalence of multidrugresistant Gram-negative bacteria (MDR-GNB).

\section{Methods}

\section{Study design}

The COMBACTE-MAGNET WP5 RESCUING study is an international, multi-centre, retrospective, observational cohort study conducted between January 2015 and August 2016. It included both patients diagnosed with CUTI as the primary reason for hospitalisation and those who developed cUTI after being hospitalised for another reason between 1st January 2013 and 31st December 2014 [12, 13]. The STROBE guidelines for reporting observational studies were followed [14]. In the present study, we compared patients who had CAUTI with those who had cUTI of other sources.

\section{Setting and patients}

The study was conducted at 20 hospitals in Bulgaria, Greece, Hungary, Israel, Italy, Romania, Spain and Turkey. Patient selection was done by searching the appropriate Clinical Modification codes at discharge based on versions 9 or 10 of the International Classification of Diseases. The sample size was calculated to detect an absolute difference of $10 \%$ in the treatment failure rate between infection due to MDR and other pathogens (power $=0.83, \alpha=0.05$ ). We planned to include 50 to 60 patients per hospital to reach a total estimated sample size of $1000[15,16]$.

The inclusion criteria were based on the Food and Drug Administration guidance for cUTI [17], requiring patients to have a UTI plus at least one of the following:

1. At least one from among the following: an indwelling urinary catheter; urinary retention (at least $100 \mathrm{~mL}$ of residual urine after voiding); neurogenic bladder; obstructive uropathy (e.g., nephrolithiasis or fibrosis); renal impairment caused by intrinsic renal disease (estimated glomerular filtration rate $<60 \mathrm{~mL} / \mathrm{min}$ ); and renal transplantation; urinary tract modification (ileal loop or pouch).

2. At least one from among the following signs or symptoms: Chills or rigors associated with fever or hypothermia (temperature $>38^{\circ} \mathrm{C}$ or $<36^{\circ} \mathrm{C}$ ); flank or pelvic pain, dysuria, urinary frequency or urgency; and costovertebral angle tenderness on physical examination.

3. A urine culture with at least $10^{5}$ colony-forming units $(\mathrm{CFU}) / \mathrm{mL}$ of a uropathogen (no more than two species); or at least one blood culture growing possible uropathogens (no more than two species) with no other evident site of infection.

We excluded patients who were younger than 18 years, diagnosed with prostatitis (based on Food and Drug Administration guidance), diagnosed with pyelonephritis with normal urinary tract, had polymicrobial infections that included Candida spp. or more than two bacterial species, or who had cUTI with Candida spp. as the sole uropathogen.

\section{Data collection}

Data from eligible patients were collected from January 2015 to August 2016. For all patients, a standardised data set was collected retrospectively from electronic hospital records and input in a web-based electronic case report form (eCRF) with controlled access. The data set included details of demographic characteristics, comorbidities, infection acquisition site, signs and symptoms, laboratory and 
microbiology test results, and discharge and outcome details, including death. The follow-up period was limited to two months after hospital discharge. To ensure data quality, study sites were monitored and audited.

\section{Definitions}

Acquisition of cUTI in a medical care facility was considered hospital-acquired if it started $48 \mathrm{~h}$ or more after hospital admission. Acquisition of cUTI was considered healthcare-associated if it was detected at hospital admission or within the first $48 \mathrm{~h}$ of hospitalisation, and met any of the following criteria: the patient had received intravenous therapy, wound care or specialist nursing care at home in the previous 30 days; hospital or haemodialysis ward attendance or intravenous chemotherapy administration in the previous 30 days; hospitalisation for at least 2 of the previous 90 days; residence in a long-term care facility; underwent an invasive urinary procedure in the previous 30 days; or had a long-term indwelling urethral catheter.

We then defined cUTI as either CA-UTI or cUTI of other source (other-cUTI). The CA-UTI group comprised those with UTI related to indwelling urinary catheterisation, including long-term, short-term or intermittent catheterisation. The other-cUTI group included those with all other causes of cUTI, including the following: UTI related to anatomical urinary tract modification (including any urinary diversion procedure, nephrostomy, stent or renal transplant); UTI related to obstructive uropathy (including any obstruction intrinsic or extrinsic to the urinary tract, such as lithiasis, tumour, ureteral herniation or prostate hyperplasia); UTI related to events that do not fall under any other category (such as neurogenic bladder).

MDR was defined in accordance with the international expert proposals of Magiorakos et al., as non-susceptibility to at least one agent in three or more antimicrobial categories (extended-spectrum penicillins, carbapenems, cephalosporins, aminoglycosides and fluoroquinolones) [18]. Extensive drug-resistance (XDR) was defined as non-susceptibility to at least one agent in all but two or fewer antimicrobial categories.

Steroid therapy was defined as the administration of a dose of at least $10 \mathrm{mg}$ of prednisolone or an equivalent dose of another steroid for 30 days or more prior to the diagnosis of cUTI.

Length of hospital stay was considered in all cases from the day of diagnosis of cUTI to the day of discharge or mortality.

\section{Clinical outcomes}

The primary endpoint was 30-day mortality. The secondary endpoints were length of hospital stay, symptom improvement at 7 days of treatment, symptom recurrence at 30 days from diagnosis, and readmission rate at 60 days from discharge. We also aimed to identify the factors influencing 30-day mortality among patients with CA-UTI.

\section{Statistical methods}

Demographic, clinical and outcome data for patients in the CA-UTI and other-cUTI groups were described using appropriate statistics according to the nature and distribution of the variable. The statistical analyses were performed using version 3.5.0 of R for Windows. Statistical significance was set at a probability level of $<0.05$.

The crude and adjusted association between the presence of CA-UTI and the 30-day mortality was analysed by logistic modelling with mixed-effects that took into account the variability between centres. The patient demographics and variables associated with 30-day mortality by unadjusted analysis (Charlson index, having haematological malignancy, basal functional status, place of cUTI acquisition and reason for admission) were used for adjustment. Although these adjustment variables were initially chosen on clinical grounds, they were required to modify the coefficient of the main variable (CA-UTI) by more than 10 to remain in the model. The impact of the assessment centre was evaluated by the intra-class correlation (ICC), which measures how much of the overall variation in the outcome is explained simply by clustering. ICC ranges from 0 to 1 ; a value close to 1 indicates that patients within centers are more similar than patients between centers, and a value close to 0 indicates that patients between centers are similar.

Residues were validated graphically, and the conditions of application of the models were tested. The odds ratios (ORs) and 95\% confidence intervals (CIs) were calculated when appropriate.

\section{Results}

\section{Baseline patient characteristics}

Table 1 shows the baseline characteristics of patients in the CA-UTI and other-cUTI groups. In total, 807 episodes of cUTI were included, of which 341 (42.2\%) had CA-UTIs. The time between catheter insertion and diagnosis was $<2$ weeks in $44.6 \%$. The CA-UTI was hospitalacquired in 130 (38.1\%), healthcare-acquired in 124 (36.4\%) and community-acquired in 87 (25.5\%) cases. Among those with hospital-acquired disease, 50 (38.4\%) cases had onsets in ICU. Compared with the other-cUTI group, the CA-UTI group were more frequently male, older, admitted for a reason other than cUTI, and acquired the infection in a medical care facility. The Charlson score was also higher in the CA-UTI group.

\section{Causative agents}

Table 2 shows the most frequent aetiologies of cUTI. Compared with the other-cUTI group, the CA-UTI group more frequently had polymicrobial infection 
Table 1 Baseline characteristics of patients with CA-UTI and other-cUTI ( $n=807$ episodes)

\begin{tabular}{|c|c|c|c|}
\hline Factors & $\begin{array}{l}\text { CA-UTI } \\
(n=341)\end{array}$ & $\begin{array}{l}\text { Other cUTI } \\
(n=466)\end{array}$ & $p$-value \\
\hline Male gender, $n(\%)$ & $197(57.8 \%)$ & $222(47.6)$ & 0.006 \\
\hline Age, median (IQR) & $71(60-82)$ & $68(56.2-79)$ & 0.009 \\
\hline Diabetes mellitus, $n(\%)$ & $109(32.2)$ & $113(24.2)$ & 0.019 \\
\hline Haematological malignancy, $n$ (\%) & $8(2.3)$ & $10(2.1)$ & 0.84 \\
\hline Solid tumour, $n(\%)$ & $42(12.3)$ & $63(13.8)$ & 0.74 \\
\hline Liver disease, $n(\%)$ & $16(4.7)$ & $26(5.58)$ & 0.68 \\
\hline Urgent admission, $n(\%)$ & $285(83.6)$ & $394(84.5)$ & 0.78 \\
\hline Admission reason: & & & $<0.001$ \\
\hline Other reasons & $213(62.5)$ & $120(25.8)$ & \\
\hline CUTI & $128(37.5)$ & $346(74.2)$ & \\
\hline Admission from medical care facility, $n(\%)$ & $88(25.8)$ & $90(13.5)$ & $<0.001$ \\
\hline Charlson score, median (IQR) & $3(1-5)$ & $2(0-4)$ & $<0.001$ \\
\hline Organ transplantation, $n(\%)$ & $10(2.9)$ & $50(10.7)$ & $<0.001$ \\
\hline Immunosuppressive therapy, $n$ (\%) & $19(5.6)$ & $63(13.5)$ & $<0.001$ \\
\hline Steroid therapy, $n(\%)$ & $22(6.5)$ & $32(6.87)$ & 0.92 \\
\hline Functional capacity: Bedridden, $n$ (\%) & $91(26.7)$ & $55(11.8)$ & $<0.001$ \\
\hline Chronic renal impairment, $n(\%)$ & $72(21.2)$ & $141(30.8)$ & 0.005 \\
\hline UTI within 1 year, $n(\%)$ & $79(23.2)$ & $143(30.8)$ & 0.021 \\
\hline Antibiotic within 30 days, $n(\%)$ & $67(19.7)$ & $104(22.5)$ & 0.39 \\
\hline Acquisition of cUTI at a medical care facility, $n(\%)$ & $254(74.5)$ & $160(34.3)$ & $<0.001$ \\
\hline Urinary retention, $n(\%)$ & $70(20.5)$ & $112(24)$ & 0.27 \\
\hline Neurogenic bladder & $32(9.4)$ & $12(2.58)$ & $<0.001$ \\
\hline Obstructive uropathy & $43(12.6)$ & $166(35.8)$ & $<0.001$ \\
\hline Severity of infection: Severe sepsis/septic shock, $n$ (\%) & $49(15.4)$ & $70(15.9)$ & 0.92 \\
\hline
\end{tabular}

CA-UTI catheter-associated urinary tract infection (CA-UTI), complicated urinary tract infection due to other sources (other-cUTI). IQR Interquartile range, CUTI complicated urinary tract infection, and UTI urinary tract infection

Table 2 Causative agents for CA-UTI and other-CUTI

\begin{tabular}{llll}
\hline Causative agents & $\begin{array}{l}\text { CA-UTI } \\
(n=341)\end{array}$ & $\begin{array}{l}\text { Other cUTI } \\
(n=466)\end{array}$ & $p$ \\
\hline Bacteraemia, $n$ (\%) & $62(18.2)$ & $67(14.4)$ & 0.14 \\
Polymicrobial infection, $n(\%)$ & $72(21.1)$ & $47(10.1)$ & $<0.001$ \\
Escherichia coli, $n$ (\%) & $127(37.2)$ & $287(61.6)$ & $<0.001$ \\
Klebsiella pneumoniae, $n(\%)$ & $63(18.5)$ & $80(17.2)$ & 0.63 \\
Pseudomonas aeruginosa, $n(\%)$ & $57(16.7)$ & $36(7.7)$ & $<0.001$ \\
Proteus mirabilis, $n(\%)$ & $40(11.7)$ & $26(5.6)$ & 0.002 \\
Acinetobacter baumannii, $n(\%)$ & $22(6.5)$ & $3(0.6)$ & $<0.001$ \\
MDR-GNB, $n$ (\%) & $120(35.2)$ & $107(23)$ & $<0.001$ \\
Enterococcus, $n$ (\%) & $38(11.1)$ & $30(6.4)$ & 0.017 \\
Staphylococcus aureus, $n$ (\%) & $6(1.8)$ & $4(0.9)$ & 0.25 \\
Other microorganisms, $n$ (\%) & $55(16.1)$ & $45(9.7)$ & 0.006 \\
\hline
\end{tabular}

CA-UTI catheter-associated urinary tract infection, complicated urinary tract infection due to other sources (other-cUTI), and MDR-GNB multidrug-resistant Gram-negative bacteria
(21.1\% vs $10.1 \%, p<0.001)$, infections caused by Pseudomonas aeruginosa $(16.7 \%$ vs $7.7 \%, \mathrm{p}<0.001)$, Proteus mirabilis $(11.7 \%$ vs $5.6 \%, p<0.001)$ or Enterococcus spp. (11.1\% vs $6.4 \%, p=0.017)$. There was also a higher frequency of MDR-GNB in the CA-UTI group compared with the other-cUTI group $(35.2 \%$ vs $23 \%, p<0.001)$. The MDR-GNB resistance profiles in patients with CAUTI are shown in the Additional file 1.

\section{Clinical outcomes Primary outcome}

The 30-day mortality in the CA-UTI group was significantly higher than that in the other-cUTI group $(52 / 341$ [15.2\%] vs $28 / 466$ [6\%], $p<0.001)$. Table 3 shows the crude association between patient characteristics and 30-day mortality considering the hospital effect in the entire cohort. Even though ICC values are not far from 0 , values above 0.20 suggest that patients' characteristics within centers are more similar than patients' characteristics between centers. The unadjusted OR of CA-UTI 
Table 3 Crude association between the baseline characteristics of patients with CUTI and the 30-day mortality

\begin{tabular}{|c|c|c|c|c|}
\hline$\underline{\text { Variable }}$ & OR & $95 \% \mathrm{Cl}$ & $p$-value & $\mathrm{ICC}^{\mathrm{a}}$ \\
\hline Male gender & 0.62 & $0.39-1.01$ & 0.07 & 0.2039 \\
\hline Age & 1.03 & $1.01-1.05$ & 0.001 & 0.1871 \\
\hline Diabetes mellitus & 0.98 & $0.58-1.66$ & 0.82 & 0.1989 \\
\hline Haematological malignancy & 6.02 & $2.11-17.23$ & $<0.001$ & 0.1918 \\
\hline Solid tumour & 1.5 & $0.78-2.89$ & 0.36 & 0.2035 \\
\hline Liver disease & 1.39 & $0.5-3.83$ & 0.65 & 0.2002 \\
\hline Charlson index & 1.21 & $1.11-1.33$ & 0.001 & 0.1723 \\
\hline Organ transplant & 0.61 & $0.2-1.87$ & 0.08 & 0.1965 \\
\hline Immunosuppressive therapy & 0.7 & $0.28-1.74$ & 0.40 & 0.1962 \\
\hline Corticosteroid therapy & 1.09 & $0.45-2.65$ & 0.21 & 0.1964 \\
\hline Bedridden functional capacity & 3.13 & $1.83-5.37$ & $<0.001$ & 0.1683 \\
\hline Chronic renal impairment & 1.16 & $0.67-2.03$ & 0.23 & 0.1817 \\
\hline UTI within 1 year & 0.77 & $0.42-1.41$ & 0.11 & 0.1878 \\
\hline Antibiotic within 30 days & 0.63 & $0.32-1.23$ & 0.09 & 0.1869 \\
\hline Acquisition of CUTI in a medical care facility & 2.42 & $1.41-4.15$ & 0.001 & 0.1677 \\
\hline Urinary retention & 0.75 & $0.39-1.44$ & 0.78 & 0.2149 \\
\hline Neurogenic bladder & 1.51 & $0.6-3.79$ & 0.39 & 0.2001 \\
\hline Obstructive uropathy & 0.46 & $0.23-0.91$ & 0.05 & 0.1905 \\
\hline Severe sepsis/ septic shock & 11.7 & $6.18-22.14$ & $<0.001$ & 0.3077 \\
\hline Polymicrobial infection & 1.16 & $0.63-2.15$ & 0.28 & 0.1947 \\
\hline MDR-GNB & 1.13 & $0.66-1.93$ & 0.69 & 0.1887 \\
\hline Urgent admission & 1.15 & $0.55-2.39$ & 0.92 & 0.2006 \\
\hline Admission reason: UTI & 0.28 & $0.16-0.5$ & $<0.001$ & 0.1683 \\
\hline Admission from a medical care facility & 2.49 & $1.47-4.19$ & 0.001 & 0.2014 \\
\hline Escherichia coli & 0.67 & $0.42-1.1$ & 0.09 & 0.1946 \\
\hline Klebsiella pneumoniae & 1.05 & $0.56-1.96$ & 0.95 & 0.1989 \\
\hline Pseudomonas aeruginosa & 0.7 & $0.31-1.58$ & 0.41 & 0.1998 \\
\hline Enterococcus & 0.9 & $0.38-2.15$ & 0.91 & 0.1999 \\
\hline Proteus mirabilis & 1.58 & $0.75-3.32$ & 0.13 & 0.1947 \\
\hline Urinary catheter as infection source & 2.56 & $1.52-4.32$ & $<0.001$ & 0.1471 \\
\hline
\end{tabular}

OR odds ratio, 95\%Cl 95\% confidence interval, ICC intra-class correlation, UTI urinary tract infection, CUTI complicated urinary tract, and MDR-GNB Multidrugresistant Gram-negative bacteria

${ }^{a}$ The ICC (range 0 to 1 ) expresses the magnitude of clustering. A high ICC (generally higher than 0.1 ) means that individuals within centers are more similar than between centers

for 30-day mortality was 2.56 (95\% CI 1.52-4.32). Table 4 shows the adjusted models of mortality first in the entire cohort (Model 1), secondly in the subgroup of patients admitted for cUTI (Model 2) and thirdly in patients admitted for other conditions than cUTI but who developed cUTI during hospitalisation (Model 3). To evaluate the effect of CA-UTI on 30-day mortality, the factor presence of severe sepsis or septic shock (unadjusted OR 11.7) was not included in the models because its strong association precluded the evaluation of any other effects. After adjustment, in none of the three models the CA-UTI remained as independent predictor of 30-day mortality (OR 1.40; 95\%CI $0.77-2.54$ in
Model 1, OR 1.62; 95\% CI 0.63-4.15 in Model 2 and OR 1.24; 95\% CI 0.54-2.81 in Model 3).

Microbiology, including the presence of MDR-GNB, had no effect on 30-day mortality. The sub-analysis of 668 cases in which we could assess the adequacy of empiric antibiotic treatment failed to show that this variable affected the 30-day mortality (Additional file 1). The factors predictive of 30-day mortality in patients with CA-UTI in the entire cohort were as follows: male gender (OR 0.53; 95\% CI 0.31-0.90) as protective factor and age (OR per year 1.57; 95\% CI 1.12-2.19), having a haematological malignancy (OR 6.08; 95\% CI 1.84-20.07), Charlson index (OR 1.45 per point; 95\% CI 1.12-1.88), being bedridden 
Table 4 Adjusted logistic mixed-effects models of predictive factors for 30-day mortality

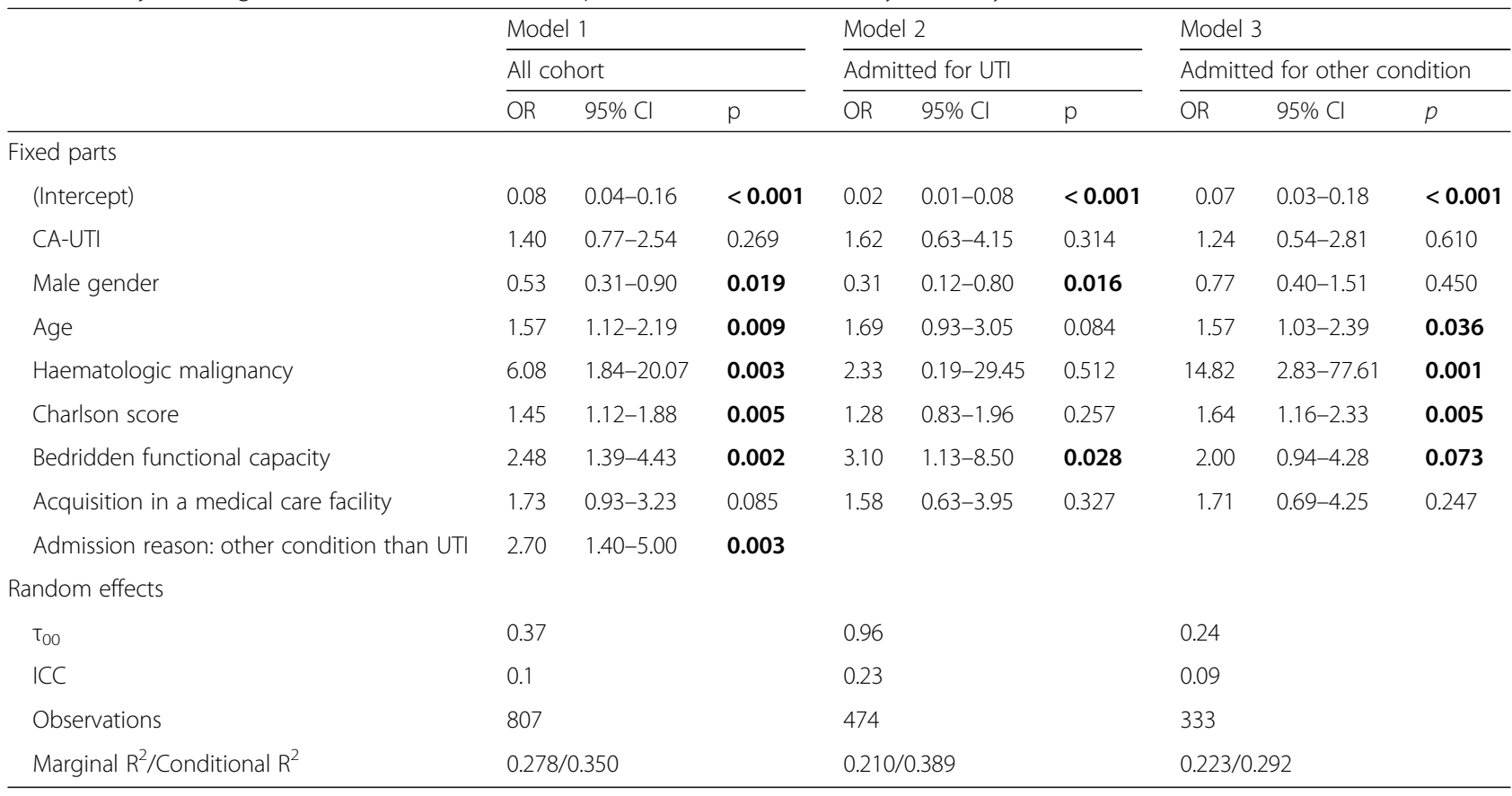

UTI urinary tract infection, OR odds ratio, 95\%Cl 95\% confidence interval, CA-UTI catheter-associated urinary tract infection, ICC intra-class correlation

(OR 2.48; 95\% CI 1.39-4.43) and being admitted for other condition than UTI (OR 2.70; 95\% CI 01.40-5.00) as risk factors. In the subgroup of patients admitted for UTI only male gender and being bedridden were independent predictors of mortality, while in the subgroup of patients admitted for other conditions than UTI predictive factors were age, having a haematological malignancy, Charlson score and being functionally bedridden.

\section{Secondary outcomes}

Patients with CA-UTI had prolonged overall length of hospital stay (median 14 [IQR 7-27] days vs 8 [IQR 514 days, $p<0.001)$ than patients with other cUTI, nevertheless, when stratifying by admission reason this increment was at the expense of the subgroup of patients admitted due to other conditions than UTI (median 20 [IQR 12-30] days in CA-UTI vs 12 [IQR 8-21] days in other cUTI, $\mathrm{p}<0.001)$. The subgroup of patients admitted for UTI showed similar length of stay regardless of the source of UTI (CA-UTI vs others), as shown in Table 5. There were no differences in other outcome variables between groups.

\section{Discussion}

In this multicentre, multinational, retrospective cohort study we observed that CA-UTI was the most frequent source of cUTI, typically involving older males with more comorbidities and MDR-GNB. The 30-day mortality of the CA-UTI group was higher than that of the other-cUTI group, but after adjusting for confounders, CA-UTI was not independently associated with 30-day mortality.

The present study included a large and recent cohort of patients with cUTI. The profile of patients with CAUTI is consistent with that reported in previous studies $[2,19]$, with these patients frequently having polymicrobial infections and MDR-GNB other than E. coli [20]. The repeated antibiotic courses and the healthcare

Table 5 Clinical outcomes in the CA-UTI and other-cUTI groups

\begin{tabular}{|c|c|c|c|}
\hline Outcomes & $\begin{array}{l}\text { CA-UTI } \\
(n=341)\end{array}$ & $\begin{array}{l}\text { Other cUTI } \\
(n=466)\end{array}$ & $p$ \\
\hline LOS of patients admitted for UTI $(n=474)^{\mathrm{a}}$ & $8(5-19)$ & $7(5-12)$ & 0.226 \\
\hline LOS of patients admitted for other condition $(n=333)^{\mathrm{a}}$ & $20(12-30)$ & $12(8-21)$ & $<0.001$ \\
\hline Improvement of symptoms at 5-7 days of treatment, $n(\%)$ & $238(70)$ & $343(73.6)$ & 0.26 \\
\hline Recurrence of symptoms at 30 days, $n(\%)$ & $28(8.3)$ & $42(9.1)$ & 0.69 \\
\hline Readmission at 60 days of discharge, $n(\%)$ & $56(16.4)$ & $91(19.5)$ & 0.25 \\
\hline
\end{tabular}

CA-UTI catheter-associated urinary tract infection, LOS length of stay, CUTI complicated urinary tract infection, IQR interquartile range

a Length of stay calculated from diagnosis of CUTI to discharge from hospital 
environment to which these patients are exposed both increase the risk of acquiring MDR-GNB strains.

Our CA-UTI group had higher 30-day mortality rates than our other-cUTI group. However, the adjusted models of mortality in the entire cohort and in the subgroups of patients according to admission reason accounting for variation between hospitals and confounding factors did not show an association between 30-day mortality and presence of CA-UTI. Nevertheless, OR of CA-UTI in patients admitted for other condition than UTI was lower than of patients admitted due to UTI, indicating that in the latter group comorbidities had the greatest impact on mortality. The presence of MDR-GNB or adequate empirical antibiotic treatment did not influence mortality in patients with CA-UTI. It should be noted that patients with severe sepsis or septic shock were excluded from these analyses because this factor strongly affects mortality and would have precluded evaluation of the effect of CA-UTI.

The relationship between CA-UTI and mortality risk in previous studies varies significantly. One study of trauma patients admitted to a single institution showed a significant association between mortality and CA-UTI, although this was mostly observed to be associated with increasing age [11]. Another study involving patients following cardiac surgery showed 30-day mortalities of 10.9 and $3.2 \%$ in patients who developed CA-UTIs or other cUTIs, but none of these deaths were directly attributed to the cUTI. Multivariate analysis discarded an association between developing a CA-UTI and mortality. Nonetheless, the authors argued that efforts to reduce CA-UTI would be worthwhile in this population by improving their management. Similar results were found in a study in which patients with CA-UTI in intensive care unit and general ward settings both had significantly higher mortality than those without CA-UTI; but, these results did not remain significant after adjustment [10].

Chant et al. [21] performed a meta-analysis of 11 observational case-control studies assessing the risk of mortality associated with CA-UTI among critically ill patients. They found no association between CA-UTI and 30-day mortality after adjusting for confounders, but did find an association between CA-UTI and length of stay, which was increased. We observed longer stays after UTI diagnosis among patients with CA-UTI than among patients without CA-UTI, mainly at the expense of those admitted for other condition than UTI. It's probable that the greater comorbidities in these patients lead to a more protracted clinical course of UTI.

As previously reported, we showed that administering adequate empiric antibiotic treatment to patients with CA-UTI made no difference to outcomes. When we also consider the role of MDR-GNB in this population, it has been suggested that treatment should be delayed until the results of sensitivity tests are available [22]. However, empiric treatment should still be initiated as soon as possible to avoid adverse outcomes in patients presenting with severe sepsis or septic shock.

The present study has a number of limitations that should be acknowledged. Notably, as with any retrospective observational study, there is a potential for residual confounding from factors that can influence 30day mortality but that were not evaluated. Nevertheless, our results are reinforced by the large-scale multi-centre design and the fact that we considered possible differences in outcomes between hospitals. By including patients with different characteristics and from different countries increases the generalisability of our data. In addition, the presence of competing events that could modify outcomes was ruled out.

\section{Conclusions}

Patients with CA-UTI tend to be older and to have greater morbidity and mortality than patients with cUTI of other sources. Despite this, the 30-day mortality does not appear to be directly related to the presence of CA-UTI.

\section{Supplementary information}

Supplementary information accompanies this paper at https://doi.org/10. 1186/s13756-019-0656-6.

Additional file 1. Antimicrobial resistance profile of Gram-negative bacteria and adjusted logistic mixed-effects model of predictive factors of 30-day mortality (with data about adequacy of empiric antibiotic treatment) in patients with catheter-associated urinary tract infection

\section{Abbreviations}

CA-UTI: catheter-associated urinary tract infections; CFU: colony-forming units; Cl: confidence interval; CUTI: complicated urinary tract infections; eCRF: electronic case report form; ICC: intra-class correlation; LTCF: long-term care facilities; MDR: multidrug-resistance; MDR-GNB: multidrug-resistant Gram-negative bacteria; OR: Odds ratio; other-cUTI: cUTI of other source; US: United States; UTI: urinary tract infections; XDR: Extensive drug-resistance

\section{Acknowledgements}

COMBACTE-MAGNET, RESCUING Study Group members: Tanya Babitch, Dora Tancheva, Rossitza Vatcheva-Dobrevska, Sotirios Tsiodras, Emmanuel Roilides, Istvan Várkonyi, Judit Bodnár, Aniko Farkas, Yael Zak-Doron, JoanM Vigo,

Yehuda Carmeli, Emanuele Durante Mangoni, Cristina Mussini, Nicola Petrosillo, Andrei Vata, Adriana Hristea, Julia Origüen, Jesus Rodriguez-Baño, Arzu Yetkin and Nese Saltoglu.

\section{Authors' contributions}

$A G, L L$ and MP conceived and designed the study. AG, JC and MP were major contributors in writing the manuscript. ES, LL, IW, CV, CV, IA participated in the design of the study and coordination and helped to draft the manuscript. CT performed the statistical analyses. All authors have read and approved the final manuscript before submission. The research leading to these results was conducted as part of the COMBACTE-MAGNET consortium. For further information please refer to <Www.COMBACTE.com>.

\section{Funding}

This research project receives support from the Innovative Medicines Initiative Joint Undertaking under grant agreement $n^{\circ} 115737$ resources of which are composed of financial contribution from the European Union Seventh Framework Programme (FP7/2007-2013) and EFPIA companies in kind contribution. 


\section{Availability of data and materials}

The datasets used and/or analysed during the current study are available from the corresponding author on reasonable request.

\section{Ethics approval and consent to participate}

This study was approved by the local research ethics committee of each site. The processing of personal data was anonymised and complied with both local data protection legislations and with the European Union Data Protection Directive (95/46/EC). The need for patient consent was waived by our ethics committee because no individual data was to be published and the patients underwent no interventions.

\section{Consent for publication}

Not applicable.

\section{Competing interests}

Author IA, CV, IW and CV belong to EFPIA (European Federation of Pharmaceutical Industries and Association) member companies in the IMI JU and costs related to their part in the research were carried by the respective company as in kind contribution under the IMI JU scheme. All other authors declare no potential conflicts.

\section{Author details}

'Department of Infectious Diseases, Hospital Universitari de Bellvitge-Institut d'Investigació Biomèdica de Bellvitge (IDIBELL), Institut Català de la Salut (ICS-HUB), Barcelona, Spain. ${ }^{2}$ Corporació Sanitària Parc Taulí, Barcelona, Spain. ${ }^{3}$ Spanish Network for Research in Infectious Diseases (REIPI RD12/0015), Instituto de Salud Carlos III (ISCIII), Madrid, Spain. ${ }^{4}$ University of Barcelona, Barcelona, Spain. ${ }^{5}$ Department of Medicine E, Beilinson Hospital, Rabin Medical Center, Petah-Tiqva; and Sackler Faculty of Medicine, Tel-Aviv University, Tel-Aviv, Israel. ' Institut d'Investigació Biomèdica de Bellvitge (IDIBELL) and Department of Basic Sciences, Universitat Rovira i Virgili, Tarragona, Spain. ${ }^{7}$ Institute of Medical Biometry and Statistics, University of Freiburg, Freiburg, Germany. ${ }^{8}$ AiCuris Anti-infective Cures GmbH, Wuppertal, Germany. ${ }^{9}$ Department of Medical Microbiology, Southmead Hospital, North Bristol NHS Trust, Bristol, UK. ${ }^{10}$ Julius Center for Health Sciences and Primary Care, University Medical Center Utrecht, Utrecht, Netherlands.

Received: 19 August 2019 Accepted: 20 November 2019

Published online: 03 December 2019

\section{References}

1. Flores-Mireles AL, Walker JN, Caparon M, Hultgren SJ. Urinary tract infections: epidemiology, mechanisms of infection and treatment options. Nat Rev Microbiol. 2015;13(5):269-84.

2. Nicolle LE. Urinary catheter-associated infections. Infect Dis Clin N Am. 2012; 26:13-27.

3. Chenoweth C, Saint S. Preventing catheter-associated urinary Tract infections in the intensive care unit. Crit Care Clin. 2013;29:19-32.

4. Saint S, Trautner BW, Fowler KE, Colozzi J, Ratz D, Lescinskas E, et al. A multicenter study of patient-reported infectious and noninfectious complications associated with indwelling urethral catheters. JAMA Intern Med. 2018;178:1078.

5. Tominaga GT, Dhupa A, McAllister SM, Calara R, Peters SA, Stuck A. Eliminating catheter-associated urinary tract infections in the intensive care unit: is it an attainable goal? Am J Surg. 2014;208:1065-70.

6. Centers for Disease Control and Prevention. NATIONAL AND STATE HEALTHCARE ASSOCIATED INFECTIONS PROGRESS REPORT 2015. Available from: https://www.cdc.gov/HAl/pdfs/progress-report/hai-progress-report-2 015.pdf. Accessed 5 May 2019.

7. Carmeli Y, Armstrong J, Laud PJ, Newell P, Stone G, Wardman A, et al. Ceftazidime-avibactam or best available therapy in patients with ceftazidime-resistant Enterobacteriaceae and Pseudomonas aeruginosa complicated urinary tract infections or complicated intra-abdominal infections (REPRISE): a randomised, pathogen-directed, phase 3 study. Lancet Infect Dis. 2016;16:661-73.

8. Hsueh P-R, Hoban DJ, Carmeli Y, Chen S-Y, Desikan S, Alejandria M, et al. Consensus review of the epidemiology and appropriate antimicrobial therapy of complicated urinary tract infections in Asia-Pacific region. J Inf Secur. 2011;63:114-23.
9. Vallejo-Torres L, Pujol M, Shaw E, Wiegand I, Vigo JM, Stoddart M, et al. Cost of hospitalised patients due to complicated urinary tract infections: a retrospective observational study in countries with high prevalence of multidrug-resistant gram-negative bacteria: the COMBACTE-MAGNET, RESCUING study. BMJ Open. 2018;8:e020251.

10. Clec'h C, Schwebel C, Français A, Toledano D, Fosse J-P, Garrouste-Orgeas $M$, et al. Does catheter-associated urinary tract infection increase mortality in critically ill patients? Infect Control Hosp Epidemiol. 2007;28:1367-73.

11. Monaghan SF, Heffernan DS, Thakkar RK, Reinert SE, Machan JT, Connolly MD, et al. The development of a urinary Tract infection is associated with increased mortality in trauma patients. J Trauma Inj Infect Crit Care. 2011;71:1569-74.

12. Shaw E, Addy I, Stoddart M, Vank C, Grier S, Wiegand I, et al. Retrospective observational study to assess the clinical management and outcomes of hospitalised patients with complicated urinary tract infection in countries with high prevalence of multidrug resistant gram-negative bacteria (RESCUING). BMJ Open. 2016;6:e011500.

13. Eliakim-Raz N, Babitch T, Shaw E, Addy I, Wiegand I, Vank C, et al. Risk factors for treatment failure and mortality among hospitalised patients with complicated urinary Tract infection: a multicentre retrospective cohort study, RESCUING Study Group. Clin Infect Dis. 2019 Jan 1;68(1):29-36.

14. von Elm E, Altman DG, Egger M, Pocock SJ, Gøtzsche PC, Vandenbroucke $J P$. The strengthening the reporting of observational studies in epidemiology (STROBE) statement: guidelines for reporting observational studies. Int J Surg. 2014;12:1495-9.

15. International Classification of Diseases, Ninth Revision, Clinical Modification, ICD-9-CM. Available at: https://www.cdc.gov/nchs/icd/icd9cm.htm. Accessed 14 March 2019

16. International Classification of Diseases, Tenth Revision, Clinical Modification, ICD-10-CM. Available at: https://www.cdc.gov/nchs/icd/icd10cm.htm. Accessed 14 March 2019.

17. Tract CU. Guidance for industry complicated urinary Tract infections : developing drugs for treatment. 2012. Available from: https://www.fda.gov/ downloads/Drugs/Guidances/ucm070981.pdf. Accessed 5 May 2019.

18. Magiorakos A-P, Srinivasan A, Carey RB, Carmeli Y, Falagas ME, Giske CG, et al. Multidrug-resistant, extensively drug-resistant and pandrug-resistant bacteria: an international expert proposal for interim standard definitions for acquired resistance. Clin Microbiol Infect. 2012;18:268-81.

19. Nicolle LE. Catheter-related urinary Tract infection: practical Management in the Elderly. Drugs Aging. 2014;31:1-10.

20. Barbadoro P, Labricciosa FM, Recanatini C, Gori G, Tirabassi F, Martini E, et al. Catheter-associated urinary tract infection: role of the setting of catheter insertion. Am J Infect Control. 2015:43:707-10.

21. Chant C, Smith OM, Marshall JC, Friedrich JO. Relationship of catheterassociated urinary tract infection to mortality and length of stay in critically ill patients: a systematic review and meta-analysis of observational studies. Crit Care Med. 2011:39:1167-73.

22. Babich T, Zusman O, Elbaz M, Ben-Zvi H, Paul M, Leibovici L, et al. Empirical antibiotic treatment does not improve outcomes in catheter-associated urinary Tract infection: prospective cohort study. Clin Infect Dis. 2017;65:1799-805.

\section{Publisher's Note}

Springer Nature remains neutral with regard to jurisdictional claims in published maps and institutional affiliations.

Ready to submit your research? Choose BMC and benefit from:

- fast, convenient online submission

- thorough peer review by experienced researchers in your field

- rapid publication on acceptance

- support for research data, including large and complex data types

- gold Open Access which fosters wider collaboration and increased citations

- maximum visibility for your research: over $100 \mathrm{M}$ website views per year

At $\mathrm{BMC}$, research is always in progress.

Learn more biomedcentral.com/submissions 